\title{
Clinical presentation and therapeutic management of venous thrombosis in young children: a retrospective analysis
}

Anthony Chan ${ }^{1}$, Anthonie W. A. Lensing 2,3,18*, Dagmar Kubitza ${ }^{2,3}$, Grahaem Brown ${ }^{4}$, Dolores Elorza ${ }^{5}$, Marta Ybarra ${ }^{5}$, Jacqueline Halton ${ }^{6}$, Sebastian Grunt ${ }^{7}$, Gili Kenet $^{8}$, Damien Bonnet ${ }^{9}$, Amparo Santamaria $^{10}$, Paola Saracco ${ }^{11}$,

Tina Biss ${ }^{12}$, Francesco Climent ${ }^{13}$, Philip Connor ${ }^{14}$, Joseph Palumbo ${ }^{15}$, Kirstin Thelen ${ }^{2,3}$, William T. Smith ${ }^{2,3}$,

Amy Mason ${ }^{2,3}$, Ivet Adalbo ${ }^{2,3}$, Scott D. Berkowitz ${ }^{2,3}$, Eva Hurst ${ }^{4}$, Jeroen van Kesteren ${ }^{4}$, Guy Young ${ }^{16}$

and Paul Monagle ${ }^{17}$

\begin{abstract}
Background: Venous thromboembolism (VTE) in young children is not well documented.

Methods: Clinicians from 12 institutions retrospectively evaluated the presentation, therapeutic management, and outcome of VTE in children younger than 2 years seen in 2011-2016. Feasibility of recruiting these children in EINSTEIN-Jr. phase III, a randomized trial evaluating rivaroxaban versus standard anticoagulation for VTE, was assessed.

Results: We identified 346 children with VTE, of whom 227 (65.6\%) had central venous catheter-related thrombosis (CVC-VTE), 119 (34.4\%) had non-CVC-VTE, and 156 (45.1\%) were younger than 1 month. Of the 309 children who received anticoagulant therapy, $86(27.8 \%)$ had a short duration of therapy (i.e. $<6$ weeks for CVC-VTE and $<3$ months for non-CVC-VTE) and 17 (5.5\%) had recurrent VTE during anticoagulation $(n=8,2.6 \%)$ or shortly after its discontinuation $(n=9,2.9 \%)$. A total of $37(10.7 \%)$ children did not receive anticoagulant therapy and $4(10.5 \%)$ had recurrent VTE.

The average number of children aged $<0.5$ years and $0.5-2$ years who would have been considered for enrolment in EINSTEIN-Jr is approximately 1.0 and 0.9 per year per site, respectively.

Conclusions: Young children with VTE most commonly have CVC-VTE and approximately one-tenth and one-fourth received no or only short durations of anticoagulant therapy, respectively. Recurrent VTE rates without anticoagulation, during anticoagulation or shortly after its discontinuation seem comparable to those observed in adults. Short and flexible treatment durations could potentially increase recruitment in EINSTEIN-Jr. phase III.
\end{abstract}

Keywords: Venous thromboembolism (VTE), Pediatric trial, Rivaroxaban, Direct oral anticoagulant (DOAC/NOAC), Anticoagulation, Registry

\section{Background}

Pediatric anticoagulant guidelines for venous thromboembolism (VTE) are mainly based on extrapolation from trials in adults, with low levels of evidence from the pediatric population. However, anecdotal evidence in neonates resulted in suggested (Grade $2 \mathrm{C}$ recommendations) treatment durations of between 6 weeks and

\footnotetext{
* Correspondence: anthonie.lensing@bayer.com

2Bayer AG, Wuppertal, Germany

${ }^{3}$ Bayer U.S., LLC, Whippany, USA

Full list of author information is available at the end of the article
}

3 months for both central venous catheter-related VTE (CVC-VTE) and other VTE (e.g. renal vein thrombosis or cerebral sinus venous thrombosis) [1]. In clinical practice, shorter durations of treatment (i.e. $<3$ months) are frequently used in children beyond the neonatal age group, presumably related to the difficulties in delivering anticoagulation using currently available drugs.

Performing clinical studies in young children with VTE is challenging for a variety of reasons. First, VTE is a relatively rare condition in children with reported incidences being approximately 100 times lower than in 
adults, requiring large and expensive collaborative efforts to recruit even a low number of children [2-8]. Second, young children with VTE represent a sick population since their VTE is often hospital acquired, largely due to more aggressive treatments of serious and life-threatening comorbidities [9]. Third, international restrictions on blood volumes that can be drawn for purposes other than routine medical care, limit participation of those with a low bodyweight [10]. Finally, parental consent is often difficult to obtain in young children with acute serious conditions in combination with a short decision window.

The EINSTEIN-Jr phase III study (clinicaltrials.gov NCT02234843) is a randomized study comparing the efficacy and safety of bodyweight-adjusted rivaroxaban with standard anticoagulation for the treatment of acute VTE in children [11]. The study started in 2014 with children aged 12-18 years and, in a staggered approach, continued with those aged 6-12 years, and 2-6 years. Study treatment is given for 3 months with the option to continue treatment in 3 months increments, up to a total duration of 12 months, with a repeat imaging test scheduled at month 3. During the last phase of the EINSTEIN-Jr. study, recruitment will be opened for neonates and infants younger than 2 years.

In this retrospective analysis, we describe the presentation, therapeutic management, and outcomes observed in children younger than 2 years who received care for VTE in recent years. In addition, we assessed their potential eligibility for enrolment into the EINSTEIN-Jr trial.

\section{Methods}

A group of EINSTEIN-Jr. investigators from 12 institutions in North America, Europe and Israel collected data from their institutions for the period 2011-2016 for children younger than 2 years with VTE and described the diagnosis of VTE at the time of presentation, if anticoagulant therapy was instituted, and the type of therapy. In addition, they assessed if children had developed recurrent VTE.

The potential eligibility for the EINSTEIN-Jr study of children with VTE seen in 2016 was done by determining the proportion of children who would have passed the screen of inclusion and exclusion criteria, as proposed in the protocol (Table 1). Then, investigators indicated whether or not they would have considered each child for inclusion into the study. This question was intended to capture additional factors not specified by the eligibility criteria, such as the child being considered too ill, or because the child was discharged to a remote location.

Data that was collected from the records included year of diagnosis, site of venous thrombosis, gestational age and bodyweight at birth, age at diagnosis, type of anticoagulant treatment (if given), and occurrence of recurrent VTE. For the purposes of this study, short duration treatment was defined a priori as $<6$ weeks for those CVC-VTE and $<3$ months for those with non-CVC-VTE. In addition, other details available to address the set of eligibility criteria were collected. To better describe factors which may contribute to children being rendered ineligible for participation in EINSTEIN-Jr, the cumulative loss of children for the year 2016 is depicted in a funnel diagram. For the latter analysis, children for whom missing data was reported were assumed to have fulfilled criteria.

The search strategies to identify children with VTE varied between the sites and consisted of review of anticoagulation prescription records from the hospital pharmacy database, the investigator's referral records, discharge letters, and department's patient records. At a single site, data was collected for the first 2 months of each year and, therefore, does not represent a complete sample over the time period 2011-2016.

The study was conducted by Competitive Drug Development International Ltd. (CDDI), London, United Kingdom in collaboration with Bayer AG. The protocol was approved by the Institutional Review Board or Ethics Committee of each participating center, when required.

\section{Data handling}

De-identified data was retrieved and entered into a validated electronic data capture system at the investigator site and, once queries had been clarified, downloaded into an Excel workbook. Results were calculated as percentages.

\section{Results}

\section{Patients}

A total of 346 children younger than 2 years were identified of whom 227 (65.6\%) had CVC-VTE and 119 (34.4\%) had non-CVC-VTE (Table 2). The age distribution of these children is shown in Fig. 1. VTE was most prevalent during the first months of life with 156 (45.1\%) being younger than 1 month. Most children were diagnosed with VTE in neonatal or pediatric intensive or high care units $(91 \%$ of children $<0.5$ years and $67 \%$ of children aged $0.5-2$ years). The most frequently involved anatomical sites in the CVC-VTE group were the extremities, caval vein or lungs $(123,54.2 \%)$, jugular or subclavian vein $(61,26.9 \%)$, and the heart $(24,10.6 \%$; Table 2). The most frequently involved anatomical sites in the non-CVC-VTE group were the cerebral veins and sinuses $(45,37.8 \%)$, and extremities, caval vein or lungs (27, 22.6\%; Table 2).

\section{Anticoagulant therapy}

A total of 37 (10.7\%) of the 346 children did not receive anticoagulant treatment (Table 2). Of the 309 children who had anticoagulant therapy, $282(91.3 \%)$ received low molecular weight heparin, 24 (7.8\%) unfractionated 
Table 1 Inclusion and exclusion criteria of the EINSTEIN Jr. study in children younger than 2 years as evaluated in this feasibility assessment

Eligibility criteria
Inclusion criteria for children aged <0.5 year
Confirmed VTE and initial treatment with therapeutic dosages of UFH,
LMWH or fondaparinux and requirement for anticoagulant therapy for at
least 3 months (at least 6 weeks for those with catheter-related VTE)
Gestational age at birth of at least 37 weeks

Oral, nasogastric or gastric feeding for at least 10 days

Bodyweight $\geq 2600 \mathrm{~g}$

Inclusion criteria for children aged $0.5-2$ years

Confirmed VTE and initial treatment with therapeutic dosages of UFH, LMWH or fondaparinux and requirement for anticoagulant therapy for at least 3 months (at least 6 weeks for those with catheter-related VTE)

Exclusion criteria

Active bleeding or bleeding risk contraindicating anticoagulant therapy

Estimated glomerular filtration rate $<30 \mathrm{~mL} / \mathrm{min} / 1.73 \mathrm{~m}^{2}$ (in children $<1$ year, serum creatinine results above 97.5th percentile [24, 25]

Hepatic disease associated with either a coagulopathy leading to a clinically relevant bleeding risk, or ALT $>5 \times$ ULN

Platelet count $<50 \times 10^{9} / \mathrm{L}$

Sustained uncontrolled hypertension defined as systolic and/or diastolic blood pressure $>$ 95th age percentile [26]

Life expectancy $<3$ months

Concomitant use of strong inhibitors of both CYP3A4 and P-gp;

Concomitant use of strong inducers of CYP3A4

Gastrointestinal disease associated with impaired absorption

Hypersensitivity or any other contraindication listed in the local labeling for rivaroxaban or comparator treatment

Participation in a study with an investigational drug or medical device within 30 days prior to randomization

\section{Rationale}

Target population

Maturation of organs involved in rivaroxaban absorption and clearance depend on the gestational and postnatal age. Rivaroxaban PK variability is expected to be higher in children born preterm compared to term neonates and older children [15-21].

Literature data indicates that gastrointestinal conditions are more stable in children with a gestational age of $\geq 37$ weeks who have been on oral feeding for at least 10 days [15-21]. Rivaroxaban should be taken with food to achieve optimal absorption $[22,23]$.

Above $2600 \mathrm{~g}$ representative virtual children could be simulated with the rivaroxaban PBPK model for (term born) neonates

Target population

Potential risk factor for (increased) bleeding with any anticoagulant

Potential risk factor for bleeding with any anticoagulant

Potential risk factor for bleeding with any anticoagulant

Potential risk factor for bleeding with any anticoagulant Potential risk factor for bleeding with any anticoagulant

A priori likelihood for the child to not complete the study

Potential for increased rivaroxaban plasma concentrations to a clinically relevant degree

Potential for reduced rivaroxaban plasma concentrations Potential for reduced rivaroxaban plasma concentrations Contraindication for use of the product

Regulatory requirement

VTE denotes venous thromboembolism, UFH unfractionated heparin, LMWH low molecular weight heparin, ALT alanine aminotransferase, ULN upper limit of normal, CYP 3A4 cytochrome P450 isoenzyme 3A4, P-gp P-glycoprotein

heparin, $2(0.6 \%)$ fondaparinux, and $1(0.3 \%)$ received only therapy with vitamin $K$ antagonists, whereas 6 (1.9\%) children transitioned from heparins to vitamin $\mathrm{K}$ antagonists. Type of anticoagulant and treatment durations were similar for the various manifestations of venous thrombosis and across age groups (Tables 2 and 3).

\section{Efficacy outcomes}

Of the 309 children who received anticoagulation, 17 (5.5\%) had a symptomatic recurrent VTE (Table 4), which occurred during anticoagulation in 8 (2.6\%; CVC-VTE group, 6/199 (3.0\%); non-CVC-VTE group, $2 / 110(1.8 \%)$ ) and following discontinuation of anticoagulation in 9 (2.9\%; CVC-VTE group, $8 /$ 199 (4.0\%); non-CVC-VTE group, 1/110 (0.9\%)). Of the 37 children who were not treated with anticoagulants, 4 (10.5\%); had a recurrent VTE (all 4 were in the CVC-VTE group, 4/28 (14.3\%); non-CVC-group, $0 / 9(0 \%))$. Results were similar across age categories (data not shown). 
Table 2 Type of anticoagulant therapy given for children younger than 2 years diagnosed with VTE

\begin{tabular}{|c|c|c|c|}
\hline & $\begin{array}{l}\text { Total population, } \\
N=346\end{array}$ & $\begin{array}{l}\text { Children aged } \\
<0.5 \text { year, } N=271\end{array}$ & $\begin{array}{l}\text { Children aged } \\
0.5-2 \text { years, } N=75\end{array}$ \\
\hline CVC-VTE, n (\%) & $227(65.6)$ & $175(64.6)$ & $52(69.3)$ \\
\hline Anticoagulation, ${ }^{a} n(\%)$ & $199(88)$ & $153(87)$ & $46(88)$ \\
\hline No anticoagulation, $n(\%)$ & $28(12)$ & $22(13)$ & $6(12)$ \\
\hline Non-CVC-VTE, n (\%) & $119(34.4)$ & $96(35.4)$ & $23(30.7)$ \\
\hline Anticoagulation, ${ }^{\mathrm{a}} \mathrm{n}(\%)$ & $110(92)$ & $88(92)$ & $22(96)$ \\
\hline No anticoagulation, $n(\%)$ & $9(8)$ & $8(8)$ & $1(4)$ \\
\hline \multicolumn{4}{|l|}{ CVC-VTE } \\
\hline \multicolumn{2}{|c|}{ Extremity/caval vein thrombosis/pulmonary embolism } & 89 & 34 \\
\hline \multicolumn{2}{|l|}{ Anticoagulation, ${ }^{a} n(\%)$} & $76(85)$ & $30(88)$ \\
\hline \multicolumn{2}{|l|}{ No anticoagulation, $n(\%)$} & $13(15)$ & $4(12)$ \\
\hline \multicolumn{2}{|l|}{ Cardiac thrombosis } & 20 & 4 \\
\hline \multicolumn{2}{|l|}{ Anticoagulation, ${ }^{a} n(\%)$} & $18(90)$ & $4(100)$ \\
\hline \multicolumn{2}{|l|}{ No anticoagulation, $n(\%)$} & $2(10)$ & 0 \\
\hline \multicolumn{2}{|l|}{ Renal vein thrombosis } & 1 & 0 \\
\hline \multicolumn{2}{|l|}{ Anticoagulation, ${ }^{a} n(\%)$} & $1(100)$ & - \\
\hline \multicolumn{2}{|l|}{ No anticoagulation, $n(\%)$} & 0 & - \\
\hline \multicolumn{2}{|l|}{ Portal vein thrombosis } & 18 & 0 \\
\hline \multicolumn{2}{|l|}{ Anticoagulation, ${ }^{a} n(\%)$} & $14(78)$ & - \\
\hline \multicolumn{2}{|l|}{ No anticoagulation, $n(\%)$} & $4(22)$ & - \\
\hline \multicolumn{2}{|l|}{ Jugular/subclavian vein thrombosis } & 47 & 14 \\
\hline \multicolumn{2}{|l|}{ Anticoagulation, ${ }^{a} n(\%)$} & $44(94)$ & $12(86)$ \\
\hline \multicolumn{2}{|l|}{ No anticoagulation, $n(\%)$} & $3(6)$ & $2(14)$ \\
\hline \multicolumn{4}{|l|}{ Non-CVC-VTE } \\
\hline \multicolumn{2}{|l|}{ Cerebral venous sinus thrombosis } & 37 & 8 \\
\hline \multicolumn{2}{|l|}{ Anticoagulation, ${ }^{a} n(\%)$} & $34(92)$ & $8(100)$ \\
\hline \multicolumn{2}{|l|}{ No anticoagulation, $n$ (\%) } & $3(8)$ & 0 \\
\hline \multicolumn{2}{|c|}{ Extremity/caval vein thrombosis/pulmonary embolism } & 16 & 11 \\
\hline \multicolumn{2}{|l|}{ Anticoagulation, ${ }^{a} n(\%)$} & $14(88)$ & $11(100)$ \\
\hline \multicolumn{2}{|l|}{ No anticoagulation, $n(\%)$} & $2(13)$ & 0 \\
\hline \multicolumn{2}{|l|}{ Cardiac thrombosis } & 12 & 0 \\
\hline \multicolumn{2}{|l|}{ Anticoagulation, ${ }^{a} n(\%)$} & $12(100)$ & - \\
\hline \multicolumn{2}{|l|}{ No anticoagulation, $n$ (\%) } & 0 & - \\
\hline Renal vein thrombosis ${ }^{\mathrm{b}}$ & & 14 & 0 \\
\hline Anticoagulation, ${ }^{\mathrm{a}} \mathrm{n}(\%)$ & & $12(86)$ & - \\
\hline No anticoagulation, $n(\%)$ & & $2(14)$ & - \\
\hline Portal vein thrombosis ${ }^{b}$ & & 14 & 1 \\
\hline Anticoagulation, ${ }^{a} n(\%)$ & & $14(100)$ & 0 \\
\hline No anticoagulation, $n(\%)$ & & 0 & $1(100)$ \\
\hline Jugular/subclavian vein thrombosis & & 4 & 3 \\
\hline Anticoagulation, ${ }^{\mathrm{a}} \mathrm{n}(\%)$ & & $3(75)$ & $3(100)$ \\
\hline No anticoagulation, $n(\%)$ & & $1(25)$ & 0 \\
\hline
\end{tabular}

anfractionated heparin, low molecular weight heparin, fondaparinux or vitamin $\mathrm{K}$ antagonists. ${ }^{\mathrm{b}} \mathrm{A}$ single patient had renal and portal vein thrombosis and was therefore considered in both groups 


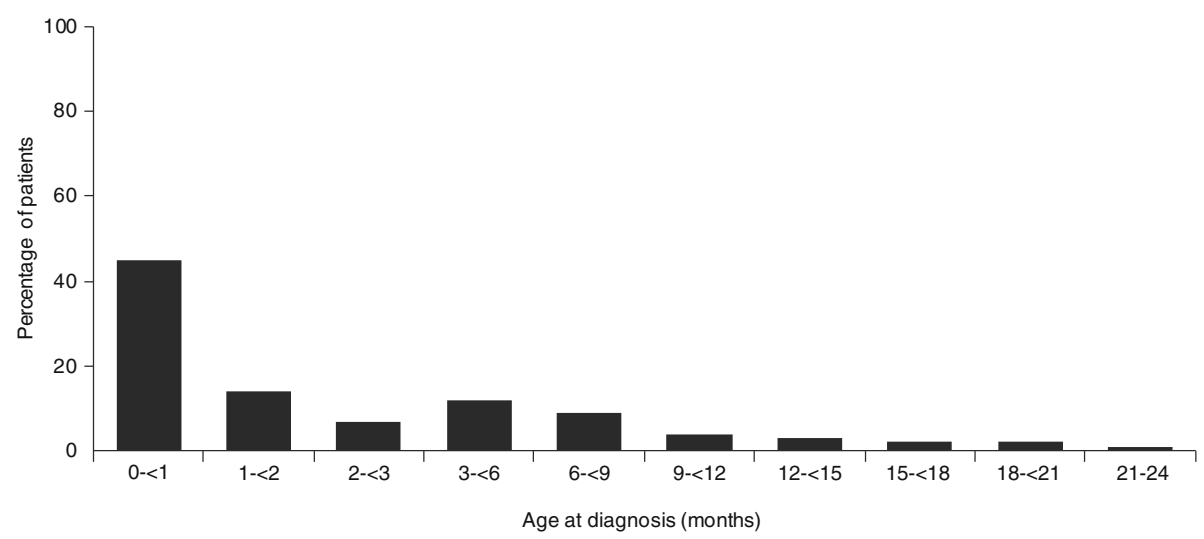

Fig. 1 Age of children younger than 2 years diagnosed in 2011-2016 ( $N=346)$

\section{Reasons for ineligibility for EINSTEIN-Jr}

The main reasons for ineligibility for children aged $<0.5$ years were a gestational age $<37$ weeks $(35.4 \%)$, a bodyweight $<2600 \mathrm{~g}(25.4 \%)$, and inadequate oral, nasogastric, or gastric feeding prior to or on the day of their diagnosis of VTE (36.5\%). Figure 2 shows the percentage of children not meeting the remaining individual inclusion and exclusion criteria of the draft EINSTEIN-Jr protocol criteria. The main reasons for ineligibility for the entire cohort of children younger than 2 years, were no anticoagulant therapy given $(11 \%)$, duration of anticoagulant therapy was too short (25\%), presence of active bleeding or high bleeding risk (20\%), gastrointestinal disease potentially associated with poor absorption (19\%), and use of potent CYP 3A4 inducers (13\%).

\section{Estimated cumulative loss of participation in EINSTEIN-Jr} For the year 2016, a total of 101 children (aged $<0.5$ years) and 35 children (aged 0.5-2 years) were evaluated using the EINSTEIN-Jr eligibility criteria (Figs. 3 and 4). Of these, $18(17.8 \%)$ and $13(37.1 \%)$ would have passed the screen of eligibility criteria of EINSTEIN-Jr., of whom 12 (11.9\%) and 11 (31.4\%) would have been considered for participation by the clinicians, respectively. This translates

Table 3 Short durations of anticoagulant treatment in relation to presenting VTE and age

\begin{tabular}{lll}
\hline & \multicolumn{2}{l}{$\begin{array}{l}\text { Duration anticoagulation shorter than } 6 \text { weeks (CVC-VTE } \\
\text { group) or shorter than } 3 \text { months (non-CVC-VTE group) }\end{array}$} \\
\cline { 2 - 3 } & $\begin{array}{l}\text { Children aged } \\
<0.5 \text { year }(n=241)\end{array}$ & $\begin{array}{l}\text { Children aged } \\
0.5-2 \text { years }(n=68)\end{array}$ \\
\hline $\begin{array}{l}\text { CVC-VTE, } \\
n=199\end{array}$ & $36 / 153(24)$ & $8 / 46(17)$ \\
$\begin{array}{l}\text { Non-CVC-VTE, } \\
n=110\end{array}$ & $35 / 88(40)$ & $7 / 22(32)$ \\
\hline
\end{tabular}

CVC-VTE denotes central venous catheter related venous thromboembolism into an average number of potentially eligible children per year per site of 1.0 and 0.9 , respectively. If a consent rate of $50 \%$ is assumed, these numbers will be 0.5 and 0.5 children per year per site, respectively.

\section{Discussion}

This study demonstrated that two-thirds of children with VTE younger than 2 years developed their VTE as a complication of the use of central catheters, with the highest incidence of VTE in the first month of life. In contradistinction to what might be expected given the current pediatric anticoagulant guidelines [1], this snapshot of contemporary clinical practice revealed that no anticoagulant therapy was given to one-tenth of children, whereas one-fourth received a short duration of anticoagulant therapy, respectively. Recurrent VTE rates without anticoagulation, during anticoagulation or shortly after its discontinuation seem comparable to those observed in adults [27-30].

The reasons behind non-adherence to the international guidelines for many children in this analysis was beyond the scope of this study, however, it is likely that it is related to the absence of any randomized, quasi-randomized, or cluster-randomized controlled trials in neonates or young children with a diagnosis of thrombosis [31]. In addition, we postulate that it could be related to the presence of serious illnesses contraindicating the use of anticoagulants, apprehension of bleeding risk in the neonatal period, the

Table 4 Recurrent VTE in relation to presence or absence of anticoagulant therapy

\begin{tabular}{lll}
\hline & CVC-VTE & Non-CVC-VTE \\
& $N=227$ & $N=119$ \\
\hline During anticoagulation, n/N (\%) & $6 / 199(3.0)$ & $2 / 110(1.8)$ \\
$\begin{array}{l}\text { Following discontinuation of } \\
\text { anticoagulants, n/N (\%) }\end{array}$ & $8 / 199(4.0)$ & $1 / 110(0.9)$ \\
No anticoagulant group, n/N (\%) & $4 / 28(14.3)$ & $0 / 9(0)$ \\
\hline
\end{tabular}




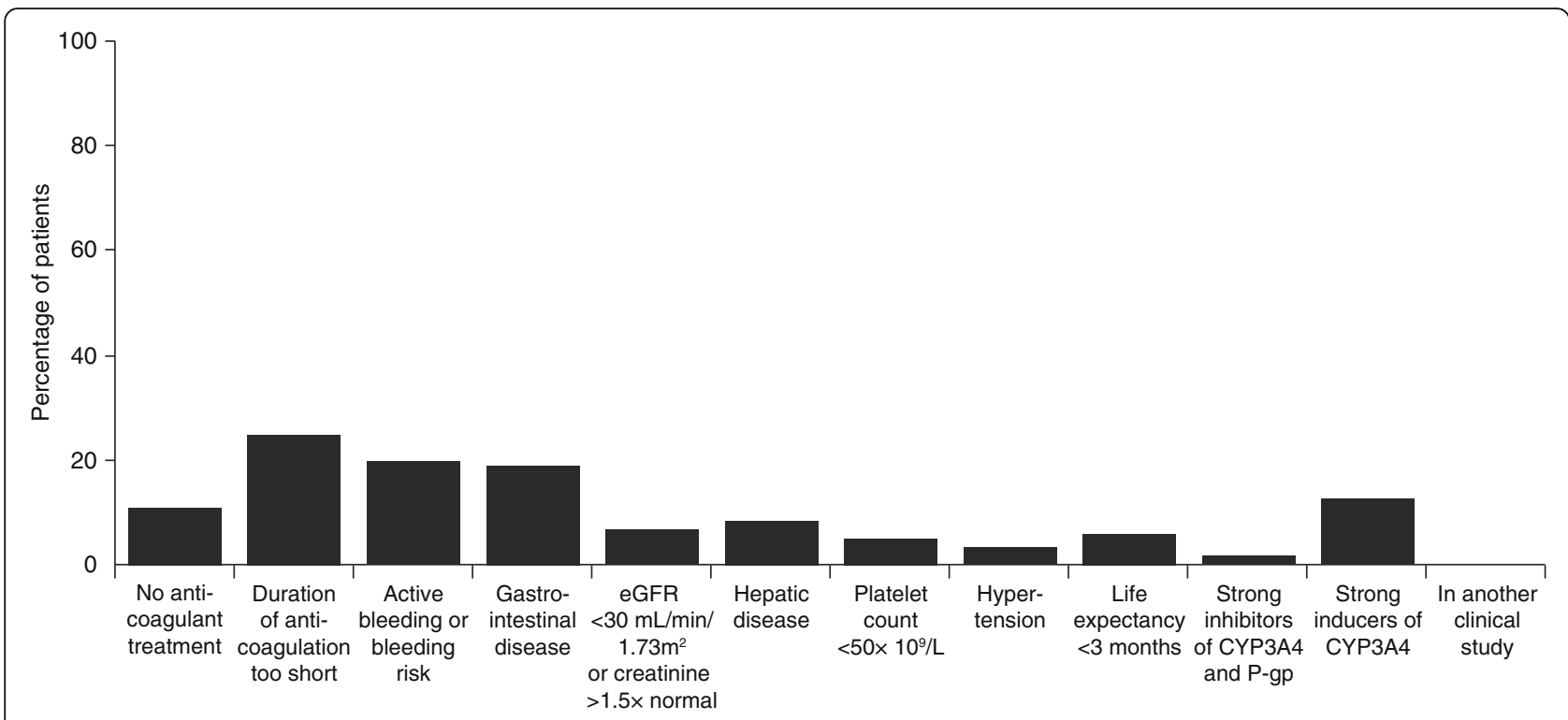

Fig. 2 Percentage of the whole population not meeting individual eligibility criteria of the draft EINSTEIN-Jr. protocol. The bars sum to more than $100 \%$ because many children would have been excluded by multiple criteria

presentation with minimal clots, and the practice of repeat ultrasound imaging to guide duration of treatment, with continuation of anticoagulation only if recanalization has failed. Whether resolution on ultrasound represents true cure, and abolition of risk of recurrence for catheter or non-catheter related VTE remains to be determined.
The most important reasons which could render children younger than 2 years ineligible for the EINSTEIN-Jr study were a gestational age of less than 37 weeks at birth, a bodyweight less than $2600 \mathrm{~g}$ and the requirement for at least 10 days of oral feeding. As a result, an inclusion rate of approximately 0.5 young child per year per site can be

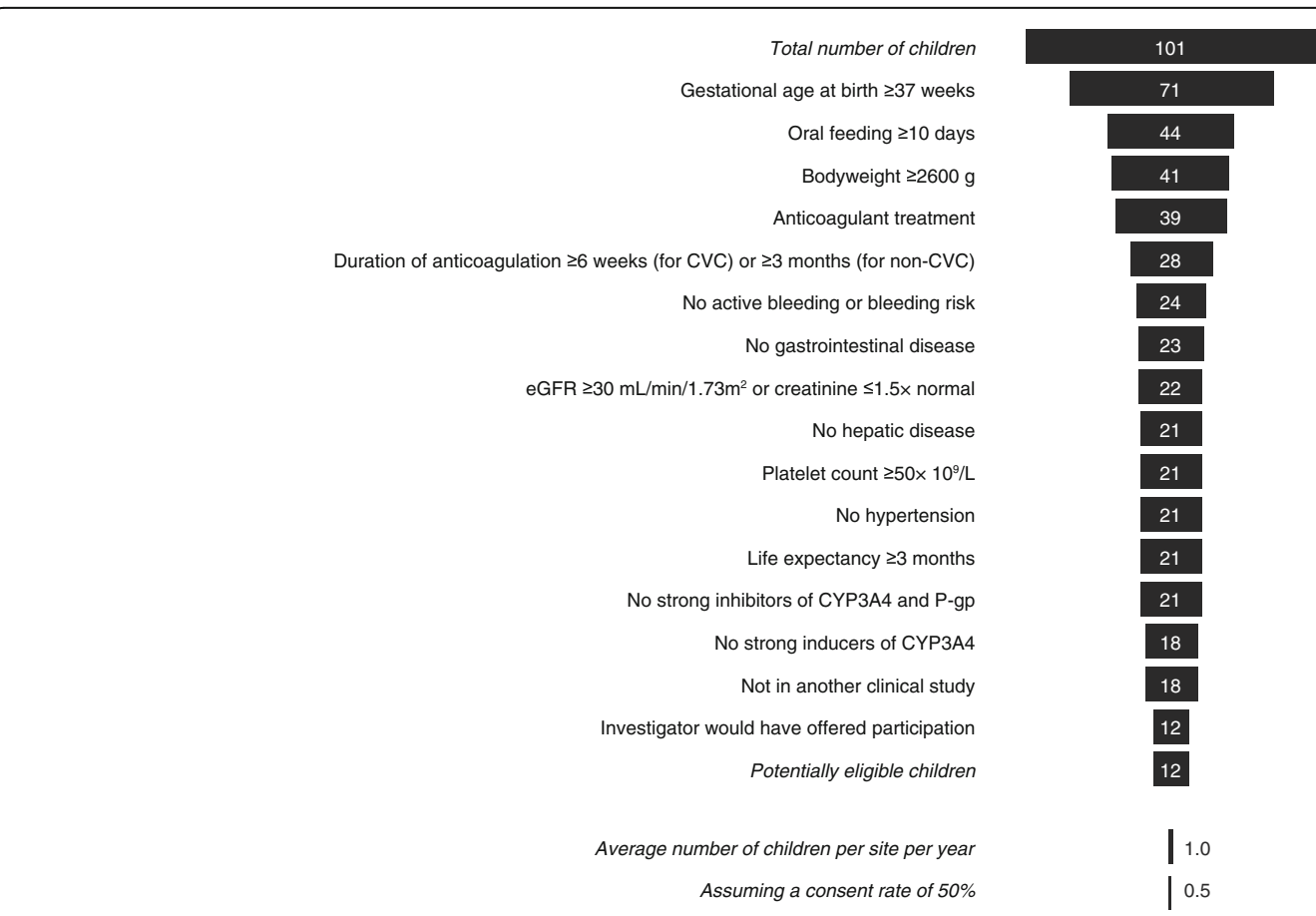

Fig. 3 Funnel showing cumulative loss of children aged $<0.5$ year due to failure to meet the proposed eligibility criteria. Missing data does not exclude the individual 


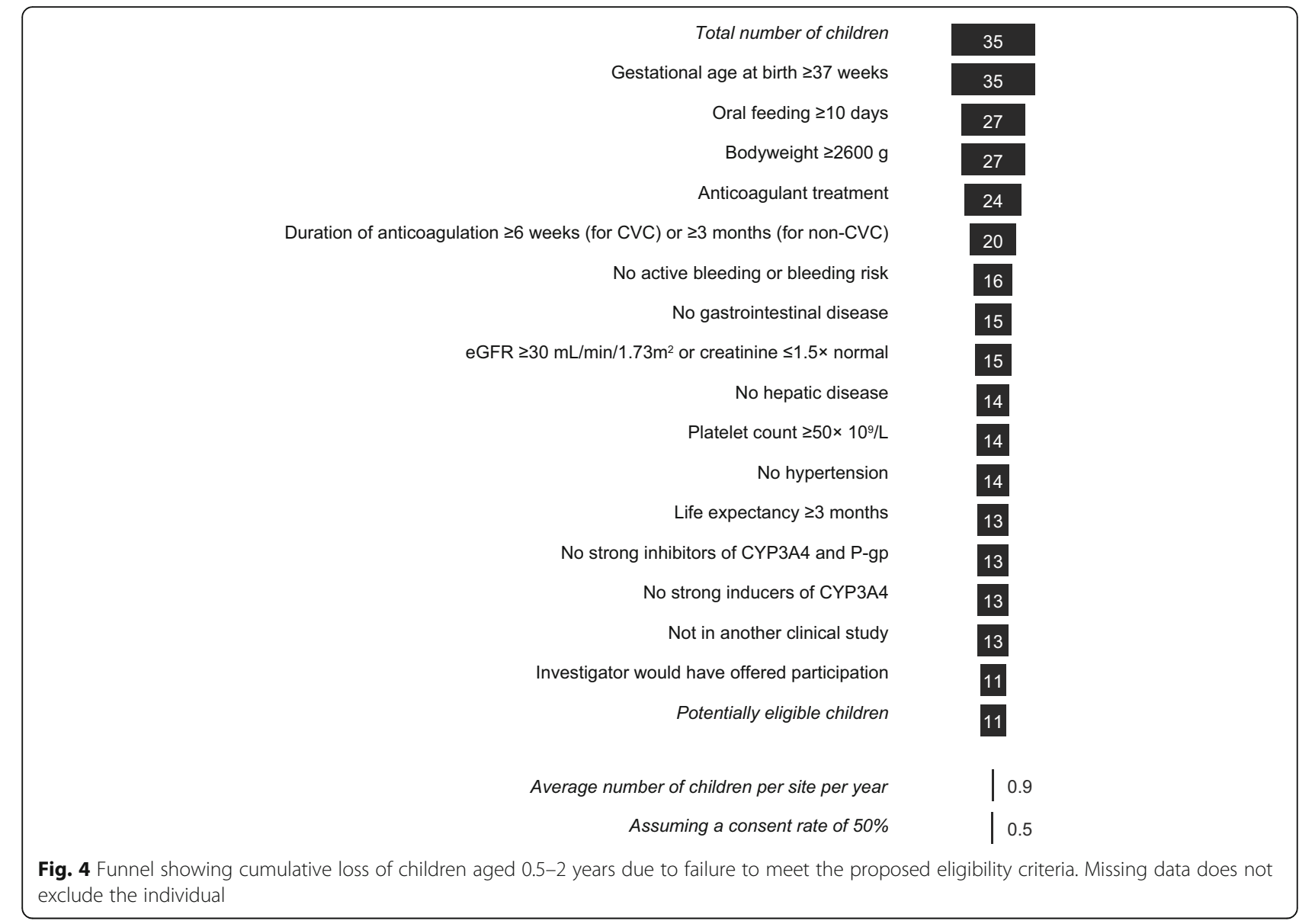

anticipated in EINSTEN-Jr. Use of short and flexible treatment durations carries potential to increase recruitment rates and appears to be in keeping with current practice for anticoagulant therapy duration, but raises the spectre of elevating expected recurrence rates.

Based on this analysis, we did not modify the screen of eligibility criteria of young children; however, we decided that young children with CVC-VTE could be treated for a minimum of 4 weeks. Although a treatment duration of less than 3 months carried the potential for increased recruitment in children younger than 2 years who had non-CVC-VTE, we decided to keep the required treatment durations at least 3 months since it is well documented that shorter periods of anticoagulation are associated with increased rates of recurrent VTE in adults [12-14]. We believe that these treatment durations will allow physicians to consider most young children with clinically significant VTE for our study. If physicians consider prolongation of anticoagulation beyond these required durations, the protocol will allow continuation of treatment for CVC-VTE in 1-month increments (up to a total duration of 3 months), and continuation of treatment for other VTE in 3-months increments (up to a total duration of 12 months). Also, repeated imaging will be done at the end of the 1-month treatment period in children with CVC-VTE and at the end of the 3-months treatment period in children with other VTE.

\section{Potential limitations}

Due to the retrospective nature of the study, we believe that our analysis may overestimate the eligibility for EINSTEIN-Jr. of young children with VTE. Although a large consecutive series of children diagnosed with VTE was identified, the physician's assessment of eligibility may have overlooked conditions and circumstances which at the time of the acute phase could have rendered the child ineligible. Furthermore, our search strategy to identify children with VTE may have been biased towards those who had received anticoagulant therapy, thereby underestimating the number of young children who did not receive anticoagulant therapy. Also, we did not capture whether anticoagulation was given in therapeutic dosages and it is, therefore, possible that some of the considered children received anticoagulation in sub-therapeutic dosages.

\section{Conclusions}

Neonates and young children with thrombosis often have CVC-VTE, and often receive no or only short durations of 
anticoagulant therapy. Recurrent VTE rates without anticoagulation, during anticoagulation or shortly after its discontinuation seem comparable to those observed in adults. Longitudinal studies to better understand the natural history of VTE in young children are urgently required. Once the population that definitely requires treatment is identified, then randomized clinical trials evaluating anticoagulant strategies are urgently needed to address the question of dose and duration, and to evaluate its benefit/risk in the treatment of VTE in young children. However, recruitment will be very challenging. Allowing short and flexible treatment durations into the EINSTEIN-Jr. phase III study could potentially increase recruitment and permit a representative population of current practice.

\section{Abbreviations}

CVC-VTE: Central venous catheter-related thrombosis; VTE: Venous thromboembolism

\section{Acknowledgements}

Data from the University Children's Hospital in Bern, Switzerland were gathered with help of the clinical trial unit PEDNET.

\section{Funding}

The study was funded by Bayer AG.

\section{Availability of data and materials}

Data are available from the authors upon reasonable request.

\section{Authors' contributions}

AC, AWAL, GY and PM created the initial draft version of this manuscript. All authors made critical revisions of the manuscript for important intellectual content, approved the final version of the manuscript for submission, and contributed to the study concept, design and implementation.

\section{Ethics approval and consent to participate}

The protocol was approved by the Institutional Review Board or Ethics Committee of each participating center, if required, and de-identified data was retrieved.

\section{Consent for publication}

See above.

\section{Competing interests}

A.C. has received consulting fees from Bayer, Daiichi Sankyo and Pfizer/BMS. A.W.A.L., D.K., K.T., W.T.S., A.M., I.A., and S.D.B. are employees of Bayer AG. G.B., E.H., and J.K. are employees of. CDDI. D.E., M.Y., J.H., S.G., G.K., D.B., A.S., P.S T.B.,F.C., P.C., J.P., G.Y. and P.M. declare they have no competing interests.

\section{Publisher's Note}

Springer Nature remains neutral with regard to jurisdictional claims in published maps and institutional affiliations.

\footnotetext{
Author details

${ }^{1}$ McMaster Children's Hospital, Hamilton, Canada. ${ }^{2}$ Bayer AG, Wuppertal, Germany. ${ }^{3}$ Bayer U.S., LLC, Whippany, USA. ${ }^{4}$ Competitive Drug Development International Ltd. (CDDI), London, UK. ${ }^{5}$ Hospital Universitario La Paz, Madrid, Spain. ${ }^{6}$ Children's Hospital of Eastern Ontario (CHEO), University of Ottawa, Ottawa, Canada. ${ }^{7}$ Division of Neuropaediatrics, Development and Rehabilitation, University Children's Hospital, University of Bern, Bern, Switzerland. ${ }^{8}$ Sheba medical center, Ramat Gan, Israel. ${ }^{9}$ Hôpital NeckerEnfants Malades, Paris, France. ${ }^{10}$ Hospital Vall d'Hebron, Barcelona, Spain. ${ }^{11}$ University Hospital, Città della Salute e della Scienza di Torino, Ospedale Infantile Regina Margherita, Torino, Italy. ${ }^{12}$ The Newcastle upon Tyne Hospitals NHS Foundation Trust, Newcastle upon Tyne, UK. ${ }^{13}$ Hospital
}

Universitario La Paz, Madrid, Spain. ${ }^{14}$ The Noah's Ark Children's Hospital for Wales, University Hospital of Wales, Cardiff, UK. ${ }^{15}$ Cincinnati Children's Hospital Medical Center, University of Cincinnati College of Medicine, Cincinnati, USA. ${ }^{16}$ Hemostasis and Thrombosis Center (HTC), Children's Hospital Los Angeles, Los Angeles, USA. ${ }^{17}$ Department of Haematology, Royal Children's Hospital, Department of Paediatrics, University of Melbourne, Murdoch Children's Research Institute, Melbourne, Australia. ${ }^{18}$ Research and Development, Thrombosis and Hematology, Building 402, room 304, Aprather Weg 18a, 42113 Wuppertal, Germany.

Received: 25 June 2018 Accepted: 19 August 2018

Published online: 01 November 2018

\section{References}

1. Monagle P, Chan AK, Goldenberg NA, Ichord RN, Journeycake JM, NowakGöttl U, Vesely SK. Antithrombotic therapy in neonates and children: antithrombotic therapy and prevention of thrombosis, 9th ed: American College of Chest Physicians Evidence-Based Clinical Practice Guidelines. Chest 2012;141(2 Suppl):e7375-e801S.

2. Raskob GE, Angchaisuksiri P, Blanco AN, et al. Thrombosis: a major contributor to global disease burden. Thromb Res. 2014;134:931-8.

3. Andrew M, David M, Adams, et al. Venous thromboembolic complications (VTE) in children: first analyses of the Canadian registry of VTE. Blood. 1994;83:1251-75.

4. van Ommen $\mathrm{CH}$, Heijboer $\mathrm{H}$, Büller $\mathrm{HR}$, et al. Venous thromboembolism in childhood: a prospective two-year registry in the Netherlands. J Pediatr. 2001:139:676-81.

5. Stein PD, Kayali F, Olson RE. Incidence of venous thromboembolism in infants and children: data from the National Hospital Discharge Survey. J Pediatr. 2004;145:563-5.

6. Sandoval JA, Sheehan MP, Stonerock CE, Shafique S, Rescorla FJ, Dalsing MC. Incidence, risk factors, and treatment patterns for deep venous thrombosis in hospitalized children: an increasing population at risk. J Vasc Surg. 2008:47:837-43.

7. Raffini L, Huang YS, Witmer C, Feudtner C. Dramatic increase in venous thromboembolism in children's hospitals in the United States from 2001 to 2007. Pediatrics. 2009;124:1001-8.

8. Chalmers EA. Epidemiology of venous thromboembolism in neonates and children. Thromb Res. 2006;118:3-12.

9. Jaffray J, Mahajerin A, Young G, Goldenberg N, Ji L, Sposto R, Stillings A, Krava E, Branchford BA. Multi-institutional registry of pediatric hospital-acquired thrombosis cases: the Children's hospital-acquired thrombosis (CHAT) project. Thromb Res. 2018;161:67-72. https://doi.org/10.1016/j.thromres.2017.11.019.

10. Howie SRC. Blood sample volumes in child health research: review of safe limits. Bull World Health Organ. 2011;89:46-53.

11. Lensing AW, Male C, Young G, et al. Rivaroxaban versus standard anticoagulation for the treatment of acute venous thromboembolism in childhood. Rationale for and design of the EINSTEIN-Jr phase III study. Blood Adv. 2018;

12. Holmgren $\mathrm{K}$, Andersson $\mathrm{G}$, Fagrell $B$, Johnsson $H$, Ljungberg B, Nilsson $E$ Wilhelmsson S, Zetterquist $S$. One-month versus six-month therapy with oral anticoagulants after symptomatic deep vein thrombosis. Acta Med Scand. 1985;218:279-84.

13. Levine MN, Hirsh J, Gent M, Turpie AG, Weitz J, Ginsberg J, Geerts W, Leclerc J, Neemeh J, Powers P. Optimal duration of oral anticoagulant therapy: a randomized trial comparing four weeks with three months of warfarin in patients with proximal deep vein thrombosis. Thromb Haemost. 1995;74:606-11.

14. Schulman $S$, Rhedin AS, Lindmarker P, Carlsson A, Lärfars G, Nicol P, Loogna E, Svensson E, Ljungberg B, Walter H. A comparison of six weeks with six months of oral anticoagulant therapy after a first episode of venous thromboembolism. Duration of anticoagulation trial study group. N Engl J Med. 1995;332:1661-5.

15. Beach RC, Menzies IS, Clayden GS, Scopes JW. Gastrointestinal permeability changes in the preterm neonate. Arch Dis Child. 1982;57:141-5.

16. Ewer AK, Durbin GM, Morgan ME, Booth IW. Gastric emptying in preterm infants. Arch Dis Child Fetal Neonatal Ed. 1994;71:F24-7.

17. Ewer AK, Durbin GM, Morgan ME, Booth IW. Gastric emptying and gastro-oesophageal reflux in preterm infants. Arch Dis Child Fetal Neonatal Ed. 1996;75:F117-21.

18. Riezzo G, Indrio F, Raimondi F, Montagna O, Salvia G, Massimo B, et al. Maturation of gastric electrical activity, gastric emptying and intestinal permeability in preterm newborns during the first month of life. Ital J Pediatr. 2009;35:6-11. 
19. Rouwet EV, Heineman E, Buurman WA, ter Riet G, Ramsay G, Blanco CE. Intestinal permeability and carrier-mediated monosaccharide absorption in preterm neonates during the early postnatal period. Pediatr Res. 2002:51:64-70.

20. Weaver LT, Laker MF, Nelson R. Intestinal permeability in the newborn. Arch Dis Child. 1984;59:236-41.

21. Taylor SN, Basile LA, Ebeling M, Wagner CL. Intestinal permeability in preterm infants by feeding type: mother's milk versus formula. Breastfeed Med. 2009:4:11-5.

22. Stampfuss J, Kubitza D, Becka M, Mueck W. The effect of food on the absorption and pharmacokinetics of rivaroxaban. Int J of Clin Pharmacol Ther. 2013;7:549-61.

23. Mueck W, Stampfuss J, Kubitza D, Becka M. Clinical pharmacokinetic and pharmacodynamic profile of rivaroxaban. Clin Pharmacokinet. 2014;53:1-16.

24. Schwartz GJ, Munoz A, Schneider MF, Mak RH, Kaskel F, Warady BA, et al. New equations to estimate GFR in children with CKD. J Am Soc Nephrol. 2009:20:629-37.

25. Boer DP, Rijke YB, Hop WC, et al. Reference values for serum creatinine in children younger than 1 year of age. Pediatr Nephrol. 2010;25:2107-13.

26. Report of the Second Task Force on Blood Pressure Control in Children -1987. Task Force on Blood Pressure Control in Children. National Heart, Lung, and Blood Institute, Bethesda, Maryland. Pediatrics. 1987;79:1-25.

27. Prins $\mathrm{MH}$, Lensing AW. Derivation of the non-inferiority margin for the evaluation of direct oral anticoagulants in the treatment of venous thromboembolism. Thromb J. 2013;11:13.

28. The EINSTEIN Investigators. Oral rivaroxaban for symptomatic venous thromboembolism. N Engl J Med. 2010;363:2499-510.

29. The EINSTEIN-PE Investigators. Oral rivaroxaban for the treatment of symptomatic pulmonary embolism. N Engl J Med. 2012;366:1287-97.

30. Prins $\mathbf{M H}$, Lensing AW, Bauersachs $\mathrm{R}$, et al. Oral rivaroxaban versus standard therapy for the treatment of symptomatic venous thromboembolism: a pooled analysis of the EINSTEIN-DVT and PE randomized studies. Thromb J. 2013:11:21.

31. Romantsik O, Bruschettini M, Zappettini S, Ramenghi LA, Calevo MG Heparin for the treatment of thrombosis in neonates. Cochrane Database Syst Rev. 2016;11:CD012185.

Ready to submit your research? Choose BMC and benefit from:

- fast, convenient online submission

- thorough peer review by experienced researchers in your field

- rapid publication on acceptance

- support for research data, including large and complex data types

- gold Open Access which fosters wider collaboration and increased citations

- maximum visibility for your research: over $100 \mathrm{M}$ website views per year

At $\mathrm{BMC}$, research is always in progress.

Learn more biomedcentral.com/submissions 